Meta

Journal des traducteurs

Translators' Journal

\title{
L'enseignement de la traduction au Mexique
}

\section{Guillermina Cuevas et Marta Pou}

Volume 35, numéro 3, septembre 1990

La traduction dans le monde hispanolusophone

URI : https://id.erudit.org/iderudit/002442ar

DOI : https://doi.org/10.7202/002442ar

Aller au sommaire du numéro

Éditeur(s)

Les Presses de l'Université de Montréal

ISSN

0026-0452 (imprimé)

1492-1421 (numérique)

Découvrir la revue

Citer cet article

Cuevas, G. \& Pou, M. (1990). L'enseignement de la traduction au Mexique. Meta, 35(3), 546-551. https://doi.org/10.7202/002442ar d'utilisation que vous pouvez consulter en ligne.

https://apropos.erudit.org/fr/usagers/politique-dutilisation/ 


\title{
L'ENSEIGNEMENT DE LA TRADUCTION AU MEXIQUE
}

\author{
Guillermina Cuevas et Marta Pou \\ El Colegio de México, México, Mexique
}

Les 9, 10 et 11 novembre 1987, a eu lieu au Colegio de México le Premier Symposium sur l'enseignement de la traduction, avec la participation de nombreux professeurs de traduction, de traducteurs professionnels et de quelques éditeurs. Cette réunion internationale, où le Canada, les États-Unis, la France et le Chili envoyèrent des représentants, a été organisée par le Programa para la formación de traductores (P.F.T.) de El Colegio de México, sous les auspices du Colegio de México, de l'Instituto Nacional de Bellas Artes, de 1'Asociación de traductores profesionales et du Bureau d'action linguistique de l'ambassade de France au Mexique.

Ces trois jours de travail intensif, sous forme de tables rondes avec une large participation des assistants, ont enrichi énormément nos connaissance sur la situation de l'enseignement de la traduction dans notre pays et provoqué certaines réflexions qui nous paraissent extrêmement importantes pour l'avenir de cette discipline au Mexique.

Bien que la situation du traducteur au Mexique, ainsi que les problèmes auxquels i1 doit faire face dans sa vie professionnelle, ne soient pas notre principal centre d'intérêt ici, il nous semble qu'il est nécessaire d'aborder brièvement ces points, qu'on ne saurait négliger lorsqu'il s'agit de connaître les besoins que devra satisfaire l'enseignement au niveau professionnel d'une discipline comme la traduction. Ces aspects ont d'ailleurs surgi constamment au long des trois jours de discussion du symposium.

Au Mexique, l'activité traductrice est encore très mal reconnue en tant que profession et se trouve trop souvent entre les mains de «traducteurs» qui se sont improvisés tels parce qu'ils ont des connaissances plus ou moins approfondies d'une langue étrangère. Les résultats sont donc souvent très médiocres, les textes publiés sont mauvais et, pour pallier cette situation, les éditeurs sont obligés d'avoir recours à un «correcteur de style» qui, dans de nombreux cas, doit se transformer en traducteur et refaire le travail. Il va sans dire que cette situation contribue à dévaloriser l'activité traductrice en tant que profession.

Il faut ajouter à cela que le traducteur non seulement ne jouit pas d'un bien grand prestige, mais encore qu'il est généralement très mal rémunéré. Il travaille donc dans des conditions difficiles, sous pression, généralement payé à la page - il y a très peu de postes fixes pour les traducteurs - et, la plupart du temps, sans pouvoir se considérer comme le vrai responsable de la version publiée; en effet, le recours au «correcteur de style» est une habitude si bien ancrée dans les maisons d'édition que même un traducteur compétent peut voir son texte modifié sans que son opinion soit prise en considération.

Car évidemment, tout ce qui précède ne veut pas dire qu'il n'y a pas au Mexique de bons traducteurs; il y en a d'excellents. Ce sont d'ailleurs certains d'entre eux qui ont été à l'origine des programmes de formation de traducteurs. La situation que nous venons de décrire est une généralisation face à laquelle nous trouvons heureusement des exceptions, mais que nous n'en devons pas moins prendre comme point de départ pour réfléchir sur la meilleure façon d'enseigner la traduction. Certes, avant qu'il y eût des programmes pour les former, il y avait de bons traducteurs, mais ce n'est qu'à travers un enseignement 
bien conçu et bien mené que nous pourrons donner toute sa force et son prestige à une discipline qui, dans beaucoup de pays, se débat encore pour être reconnue comme telle.

Voici donc le premier choix qui se pose aux responsables des programmes de formation de traducteurs : doit-on concevoir un enseignement qui prépare les étudiants en vue du marché du travail tel qu'il se présente actuellement, ou un enseignement qui les engage à le modifier? Nous pensons que l'intérêt qu'ont montré les nombreux professeurs venus d'universités de tout le Mexique pour assister au symposium prouve que c'est bien la deuxième possibilité qui a été choisie.

Voyons maintenant quelles sont les conditions dans lesquelles travaillent les formateurs de traducteurs au Mexique, en commençant par les candidats. Nous verrons à partir de là quelle sorte d'enseignement nous paraît être en mesure de former des traducteurs compétents et quels problèmes il reste à résoudre.

Un peu partout les enseignants se plaignent de ce que les étudiants écrivent de moins en moins bien leur langue maternelle; au Mexique, ce défaut atteint trop souvent un niveau critique et les élèves qui terminent le baccalauréat sont très rarement capables de rédiger correctement. Il faut ajouter que l'enseignement des langues étrangères n'est pas prioritaire aux niveaux secondaire et préparatoire, et ce n'est que très exceptionnellement qu'un élève qui commence une licence a quelques connaissances sérieuses de l'anglais ou du français (qui sont les deux langues qui figurent dans les programmes d'études secondaires).

Il faut ajouter à ces faiblesses en langues étrangères - et en langue maternelle des niveaux secondaire et préparatoire les fortes réticences exprimées par l'ensemble des institutions mexicaines d'enseignement supérieur devant l'idée d'une véritable sélection à la base, d'examens d'admission spécifiques aux diverses carrières et qui, dans le domaine qui nous intéresse, seuls donneraient toute sa valeur à une licence en traduction obtenue, après les quatre ans d'études de rigueur, par les quelques candidats à qui l'accès en aurait été permis.

Pour toutes ces raisons, nous ne croyons pas qu'une licence en traduction soit une réponse adéquate à la question posée par le niveau des études de la traduction. Cela pose, cependant, un grave problème institutionnel; en effet, au Mexique, c'est le diplôme de licence qui sanctionne l'accès à l'activité professionnelle et certains soutiennent, et c'est un argument de poids, que tant qu'il n'y aura pas une licence en traduction, comme il en existe une en médecine ou en histoire, etc., la traduction ne sera jamais considérée comme une vraie discipline au niveau professionnel.

Quant à nous, il nous semble que lancer sur le marché des professionnels sanctionnés par un diplôme universitaire mais médiocrement formés ne contribuera jamais à valoriser leur activité ni à améliorer la qualité des traductions au Mexique, et ce n'est pas chez les étudiants issus du baccalauréat que nous pensons trouver des candidats aptes à recevoir une formation de traducteurs. Ainsi, le programme pour lequel nous travaillons exige au moins quatre semestres complets d'études universitaires préalables et soumet les candidats à un rigoureux concours d'entrée centré sur les connaissances en langues étrangères et, surtout, en langue maternelle. Les étudiants acceptés sur cette base peuvent alors axer leurs deux années d'études sur la traduction proprement dite et sur les disciplines connexes.

Dans la deuxième partie de cet article, nous décrivons brièvement quelques-uns des programmes qui existent actuellement; disons tout de suite que s'il est vrai qu'il existe au Mexique des licences en traduction, les résultats n'en sont pas encore très clairs.

Pour que fonctionne un programme de formation de traducteurs, il ne faut pas seulement des élèves, il faut aussi des professeurs. Qui sont donc ces formateurs de traducteurs? Quelle est leur formation à eux? Ce sont là des questions extrêmement 
importantes, qui définissent justement l'un des problèmes à résoudre dont nous parlions plus haut.

En effet, si l'enseignement de la traduction en tant que profession est assez neuf en Europe ou au Canada, par exemple, il l'est encore plus dans notre pays.

Bien que le P.F.T. fonctionne régulièrement depuis 1974, formant entre 15 et 20 traducteurs tous les deux ans, ce n'est que très récemment que certains - très peu nombreux - de ceux qui furent «élèves de traduction» sont devenus «professeurs de traduction», et il faut bien reconnaître que la plus grande partie de ceux qui enseignent la traduction ou participent d'une manière ou d'une autre à la formation de traducteurs professionnels n'ont pas reçu de préparation spécifique ni comme professeurs, a fortiori, comme professeurs de traduction. Il s'agit parfois de traducteurs professionnels, ayant souvent reçu une formation différente - un anthropologue, un médecin ou un historien, par exemple -, qui bénéficient d'une grande expérience mais manquent souvent de connaissances approfondies en linguistique ou en stylistique. Il s'agit, enfin, assez souvent, de professeurs qui possèdent une formation en littérature ou en linguistique, parfois accompagnée d'une expérience en tant que traducteurs. L'expérience dans le domaine de la traduction a d'ailleurs été jugée comme une condition essentielle chez tous ceux qui veulent s'occuper de la formation de traducteurs.

Cet état de choses, il faut bien le dire, a donné jusqu'à maintenant d'assez bons résultats et ces professeurs de traduction «improvisés», parmi lesquels nous comptons des écrivains, traducteurs et chercheurs comme Tomás Segovia, Antonio Alatorre, Flora Botton et Monique Legros, ont contribué à former les meilleurs jeunes traducteurs qui exercent leur profession en ce moment. C'est cependant vers le futur que nous nous tournons en ce moment, vers la nécessité d'établir au Mexique la traduction comme une discipline solide. Et, pour y arriver, il nous semble essentiel de prévoir une véritable «formation de formateurs».

En effet, bien que le P.F.T. fonctionne à un niveau d'études supérieur à celui de la licence - une grande partie de nos candidats ont déjà une licence complète -, son but $n$ 'est pas encore de former des professeurs ou des chercheurs en traductologie, mais bien des traducteurs professionnels de grande qualité. Comme on pourra aisément le constater dans le programme que nous présentons à la fin de cet article, l'accent n'est pas mis sur la théorie mais sur la pratique. À ce sujet, il faut noter que les anciens élèves du P.F.T. maintenant devenus professeurs ont tous fait dans une autre institution - souvent étrangère au moins une maitrise en traduction ou en linguistique, ou bien des stages dans des disciplines connexes comme la terminologie. Nous pensons qu'il est nécessaire de prévoir au Mexique des études supérieures en traduction débouchant sur la maîtrise ou le doctorat qui pourront fournir les professeurs et les chercheurs nécessaires à l'approfondissement de la traduction en tant que discipline, non seulement en ce qui concerne la formation de professionnels compétents, mais aussi en ce qui concerne la formation des formateurs et des chercheurs en traductologie.

\section{PROGRAMMES DE FORMATION DE TRADUCTEURS}

À titre d'exemple nous présenterons maintenant, brièvement, trois programmes de formation de traducteurs dont chacun illustre un type de formation (licence, programme «post-licence», «cours de spécialisation en traduction») qui peut être considéré comme représentatif des tentatives actuelles de professionnalisation de la traduction au Mexique. Nous avons délibérément choisi de ne pas parler ici des carrières de technicientraducteur, qui existent depuis longtemps, n'exigent pas un niveau universitaire et dont les problèmes, par ailleurs, sont souvent les mêmes que ceux dont nous avons traité. 
En premier lieu, nous parlerons de la licence en traduction créée en 1985 par une université de création récente, l'Universidad Intercontinental (Mexico). Elle compte actuellement 30 élèves, qui termineront dans quelques semaines le sixième des huit semestres d'études qu'elle comporte. À la fin du huitième semestre, après présentation et soutenance d'une partie de thèse, les étudiants recevront le titre de licenciés en traduction conféré par la même université.

Nous évoquerons ensuite le seul programme «post-licence» qui existe au Mexique: le Programa para la formación de traductores du El Colegio de México, fondé en 1974 et d'où sont déjà sortis 104 traducteurs diplômés. Chaque cycle d'études dure deux ans et comporte quatre semestres; à la fin du dernier semestre El Colegio de México confère aux étudiants reçus un diplôme d'études. Nous terminons actuellement le quatrième semestre du $7 \mathrm{e}$ cycle.

Enfin, nous mentionnerons un type de programme dont l'objectif principal n'est pas la formation de traducteurs proprement dite. Il s'agit de l'un de ces «cours de traduction» qui font partie des licences en littérature ou en langues étrangères de nombreuses universités, ou bien, comme c'est le cas ici, d'un cours de spécialisation inclus dans les études de français de 1'Institut français d'Amérique latine de Mexico, dont le but principal est le perfectionnement, grâce à la traduction, du maniement de cette langue étrangère.

En ce qui concerne les programmes qui ont pour but spécifique la formation de traducteurs (nos deux premiers exemples), leur contenu varie selon la durée des études et surtout selon le profil des étudiants.

Nous ne reviendrons pas sur l'analyse du niveau de connaissance des candidats. Signalons toutefois que lorsqu'on a affaire à des étudiants qui commencent des études universitaires (cas de la licence), il faut penser davantage à «former» qu’à «compléter une formation».

Dans l'exemple que nous avons choisi, la licence en traduction de l'Universidad Intercontinental met l'accent sur la formation littéraire des étudiants qui, en outre, reçoivent quelques connaissances en théorie linguistique et théorie de la traduction.

Mais est-ce assez? Selon notre perspective, le contenu de cette licence ne serait que le point de départ d'une véritable formation de traducteurs, puisque, une fois que l'on a éveillé chez l'étudiant l'intérêt et le plaisir de travailler avec la langue comme matière première, il faudrait lui montrer que la langue littéraire n'est que l'une des façons de s'exprimer de cette matière première, et que dans sa vie professionnelle de traducteur, c'est le plus souvent la langue scientifique ou le langage technique à quoi il aura affaire. En outre, il serait nécessaire de lui montrer, voire de lui démontrer, que le plus vif et constant intérêt pour tout ce qui a lieu dans le monde est indispensable dans son métier, et cela ne sera possible que si l'étudiant est littéralement submergé dans une ambiance multidisciplinaire impossible à créer dans le cadre d'une licence traditionnelle, limitée par des contraintes administratives qui ne permettent pas de changements dictés par les besoins.

Un autre paramètre dont il faut tenir compte, c'est le nombre des étudiants et leur niveau relatif de connaissance. Au Mexique, il est en général très difficile, pour ne pas dire impossible, de pratiquer une sélection rigoureuse des candidats, puisqu'il n'existe qu'un examen général d'entrée à l'université, le même, ou presque, pour les humanités et pour les carrières techniques. Le nombre des étudiants admis ne dépend pas non plus des responsables directs de chaque carrière, mais des politiques générales de l'université. Résultat: des groupes toujours trop nombreux pour les «cours pratiques de traduction», où chaque élève doit être guidé et corrigé individuellement, des groupes dont les niveaux de connaissances en langue étrangère, et même en langue maternelle, ne sont pas homogènes, et, pis encore, n'atteignent pas le seuil nécessaire pour être initiés à la pratique de la traduction. 
On voit que la connaissance de la langue étrangère (et nous ne parlons pas de deux langues étrangères) n'étant pas une condition d'admission, la plus grande partie du temps d'étude (10 heures par semaine) est consacrée à son apprentissage.

C'est précisément pour éviter ce type de contraintes - nombre et profil d'étudiants imposés par les autorités universitaires, programme figé pendant des années et des années, production de professionnels médiocres, sans vocation et sans apprentissage pratique -, qu' El Colegio de México a décidé en 1974 de créer le Programme pour la formation des traducteurs (P.F.T.).

Cette création a été précédée par deux programmes pilotes de trois semestres chacun, d'où sortirent les premiers professeurs du P.F.T. : c'étaient, presque tous, des traducteurs professionnels, inquiets pour l'avenir de leur profession et désireux de former des jeunes traducteurs au moyen des connaissances théoriques et des techniques pratiques qui leur avaient manqué et dont ils avaient ressenti le besoin, au début de leur carrière.

Les résultats de cette inquiétude et de ces décisions sont visibles aujourd'hui : le $8^{e}$ cycle d'études du Programa para la formación de traductores commencera en septembre 1988. Des cycles passés sont sortis quelques-uns des professeurs actuels de traduction, qui, s'ils n'ont pas reçu une formation spécifique de professeurs de traduction, n'en ont pas moins une solide formation de traducteurs.

De ces cycles antérieurs sont sortis également d'excellents jeunes traducteurs, et à ce propos il faut dire qu'en 1985 deux de nos élèves ont gagné le Prix de traduction, catégorie Étudiants (offert par l'État d'Hidalgo); en 1986 une ex-étudiante - elle venait de terminer ses études au P.F.T. - a obtenu le Prix Alphonse $X$ de traduction littéraire, la plus haute récompense mexicaine dans le domaine de la traduction, offert par l'Instituto Nacional de Bellas Artes et l'Asociación de Traductores Profesionales pour sa traduction en espagnol d'un roman d'Anatole France. En 1987, c'est l'un de nos professeurs qui l'a obtenu, grâce à ses qualités de traducteur et de formateur de traducteurs.

Du point de vue universitaire, le P.F.T. est le seul programme post-licence voué exclusivement à la formation de traducteurs; parce qu'il sort du cadre traditionnel des études universitaires, il offre une souplesse qui a permis dans une certaine mesure d'introduire plusieurs modifications selon les besoins d'un groupe particulier ou les intérêts des étudiants.

Le «séminaire de travail» par exemple, n'est qu'un «artifice» qui permet de changer le contenu d'un cours sans passer par une chaîne sans fin d'autorisations administratives qui finirait par détruire n'importe quelle initiative de changement ou d'actualisation. C'est précisément ce cours-là qui nous a permis l'inclusion de thèmes comme: l'ordinateur au service du traducteur, la préparation de manuscrits pour publication, le dictaphone au service du traducteur, etc., ainsi que celle de cours supplémentaires de langue espagnole ou de stylistique comparée, quand les étudiants en ressentent le besoin.

Il y a enfin d'autres cours qui, s'ils n'ont pas pour but spécifique de former des traducteurs, s'intéressent quand même au problème pour des raisons différentes.

Dans le cas des cours dits de «spécialisation» et qui font partie des licences en littérature ou en langues étrangères, les étudiants n'acquièrent qu'une connaissance très superficielle de ce qu'est la traduction. En général il ne s'agit que de deux ou trois classes de travaux pratiques par semaine pendant les deux derniers semestres de la licence, portant presque toujours sur des textes littéraires. Quelquefois ils offrent aussi un semestre de «théorie de la traduction» (cas de l'option «spécialisation en traduction» de la licence en Langue et littérature française de la Facultad de Filosofía y Letras de l'Universidad Nacional Autónoma de México; cette «spécialisation» était offerte à côté des spécialisations en «didactique» et en «critique littéraire»). 
Il ne nous reste à citer que les cours offerts par les institutions d'enseignement de langues étrangères. Ces cours, appelés parfois de «perfectionnement de la langue», n'ont pas non plus pour but spécifique la formation de traducteurs. Ils ferment, pour ainsi dire, le cycle d'apprentissage d'une langue étrangère, et leur qualité varie avec le prestige de l'institution à laquelle ils appartiennent. Le cours de l'Institut français de l'Amérique latine (IFAL), Mexico, dont la durée est de deux semestres, introduit quelques concepts élémentaires de linguistique et quelques éléments d'histoire de la traduction, mais ne fait aucune mention des autres connaissances dont le traducteur aura besoin dans l'exercice de sa profession.

\section{CONCLUSION}

Cette vue d'ensemble montre bien que si l'enseignement de la traduction au Mexique n'est pas, dans trop de cas, au niveau où il devrait être, on est quand même sur la bonne voie. Surtout, il existe déjà chez certains responsables de la formation de traducteurs un immense intérêt pour l'extension de l'enseignement de la traduction, ainsi que pour l'approfondissement et l'analyse de ses stratégies.

Il est évident que pour un pays aussi peuplé que le nôtre, et dont la technologie, pour ne parler que d'elle, dépend dans une large mesure de l'étranger, il ne s'agit que d'un début bien modeste, qui commence à donner des fruits visibles non seulement dans la création de récompenses comme celles que nous citons plus haut, mais surtout dans le fait que la plupart des personnes qui ont reçu récemment ces prix sont de jeunes traducteurs issus d' un programme spécifique de formation de traducteurs.

Nous ne perdons pas de vue qu'il faut établir solidement l'enseignement de la traduction en tant que discipline professionnelle, et pour cela il ne suffit pas de multiplier les programmes tels qu'ils existent actuellement. Il faut entamer des recherches qui aboutiront à une meilleure connaissance des besoins, à la création de programmes adaptés au public et au marché, et avant tout à la formation de formateurs. Et pour former des formateurs il faut penser à la création de maitrises et de doctorats, par lesquels les étudiants se consacreront à des recherches concrètes et bien définies en théorie de la traduction, en linguistique, stylistique et informatique appliquées à la traduction. 